\title{
Robust Cluster Analysis via Mixtures of Multivariate $t$-distributions
}

\author{
Geoffrey J. McLachlan and David Peel \\ Department of Mathematics, University of Queensland, \\ St. Lucia, Queensland 4072, AUSTRALIA
}

\begin{abstract}
Normal mixture models are being increasingly used as a way of clustering sets of continuous multivariate data. They provide a probabilistic (soft) clustering of the data in terms of their fitted posterior probabilities of membership of the mixture components corresponding to the clusters. An outright (hard) clustering can be subsequently obtained by assigning each observation to the component to which it has the highest fitted posterior probability of belonging. However, outliers in the data can affect the estimates of the parameters in the normal component densities, and hence the implied clustering. A more robust approach is to fit mixtures of multivariate $t$-distributions, which have longer tails than the normal components. The expectation-maximization (EM) algorithm can be used to fit mixtures of $t$-distributions by maximum likelihood. The application of this model to provide a robust approach to clustering is illustrated on a real data set. It is demonstrated how the use of $t$-components provides less extreme estimates of the posterior probabilities of cluster membership.
\end{abstract}

\section{Introduction}

Finite mixtures of distributions have provided a mathematical-based approach to the statistical modeling of a wide variety of random phenomena (McLachlan and Basford (1988), Mclachlan (1999)). Because of their usefulness as an extremely flexible method of modelling, finite mixture models have continued to receive increasing attention over the years, both from a practical and theoretical point of view. For multivariate data of a continuous nature, attention has focussed on the use of multivariate normal components because of their computational convenience. They can be easily fitted iteratively by maximum likelihood (ML) via the expectation-maximization (EM) algorithm (Dempster, Laird, and Rubin (1977), McLachlan and Krishnan (1997)), as the iterates on the M-step are given in closed form.

However, for many applied problems, the tails of the normal distribution are often shorter than required. Also, the estimates of the component means and covariance matrices can be affected by observations that are atypical of the components in the normal mixture model being fitted. Hence we consider the fitting of mixtures of multivariate $t$-distributions. This provides a more robust approach to the fitting of normal mixture models, as observations that are atypical of a component are given reduced weight in the calculation of its parameters. 
Also, as the $t$-distribution provides a longer tailed alternative to the normal distribution, it tends to give less extreme estimates of the posterior probabilities of component membership.

One useful application of normal mixture models has been in the important field of cluster analysis. Besides having a sound mathematical basis, this approach is not confined to the production of spherical clusters, such as with $k$ means type algorithms that use Euclidean distance rather than the Mahalanobis distance metric which allows for within-cluster correlations between the variables in the feature vector $\boldsymbol{X}$. Moreover, unlike clustering methods defined solely in term so of the Mahalanobis distance, the normal mixture-based clustering takes into account the normalizing term $\left|\boldsymbol{\Sigma}_{i}\right|^{-1 / 2}$ in the estimate of the multivariate normal density adopted for the component distribution of $\boldsymbol{X}$ corresponding to the ith cluster. This term can make an important contribution in the case of disparate group-covariance matrices (McLachlan (1992, Chapter 2)).

Although even a crude estimate of the within-cluster covariance matrix $\boldsymbol{\Sigma}_{i}$ often suffices for clustering purposes, it can be severely affected by outliers. Hence it is highly desirable for methods of cluster analysis to be robust. By robustness, it is meant that the method is not affected significantly by small departures from the assumed model, such as the presence of outliers. The problem of providing protection against outliers in multivariate data is a very difficult problem and increases with the difficulty of the dimension of the data (Rocke and Woodruff (1997)). The related problem of making clustering algorithms more robust has received much attention recently as, for example, in McLachlan and Basford (1988, Chapter 3), De Veaux and Kreiger (1990), Campbell (1994), Davé and Krishnapuram (1996), Frigui and Krishnapuram (1996), Kharin (1996), and Rousseeuw, Kaufman, and Trauwaert (1996), and Zhuang et al. (1996), among others. In the past, there have been many attempts at modifying existing methods of cluster analysis to provide robust clustering procedures. Some of these have been of a rather ad hoc nature. The $t$-mixture model provides a sound mathematical basis for a robust method of clustering. We shall illustrate its usefulness in this context by a cluster analysis of a real data set.

\section{Normal Mixture Model}

We let $\boldsymbol{x}_{1}, \ldots, \boldsymbol{x}_{n}$ denote an observed $p$-dimensional random sample of size $n$. With a normal mixture model-based approach to drawing inferences from these data, each data point is assumed to be a realization of the random $p$-dimensional vector $\boldsymbol{X}$ with the $\boldsymbol{g}$-component normal mixture probability density function (p.d.f.),

$$
f(\boldsymbol{x} ; \boldsymbol{\Psi})=\sum_{i=1}^{g} \pi_{i} \phi\left(\boldsymbol{y} ; \boldsymbol{\mu}_{i}, \boldsymbol{\Sigma}_{i}\right),
$$

where the mixing proportions $\pi_{i}$ are nonnegative and sum to one and where $\phi\left(\boldsymbol{x} ; \boldsymbol{\mu}_{i}, \boldsymbol{\Sigma}_{i}\right)$ denotes the multivariate normal p.d.f. with mean (vector) $\boldsymbol{\mu}$ and covariance matrix $\boldsymbol{\Sigma}$. Here $\boldsymbol{\Psi}=\left(\pi_{1}, \ldots, \pi_{g-1}, \boldsymbol{\theta}^{T}\right)^{T}$, where $\boldsymbol{\theta}=\left(\boldsymbol{\theta}_{1}^{T}, \ldots, \boldsymbol{\theta}_{g}^{T}\right)^{T}$ 
and where $\boldsymbol{\theta}_{i}$ contains the elements of $\boldsymbol{\mu}_{i}$ and the distinct elements of $\boldsymbol{\Sigma}_{i}(i=$ $1, \ldots, g)$.

\section{Multivariate $t$-Distribution}

Consider the multivariate normal p.d.f. $\phi(\boldsymbol{x} ; \boldsymbol{\mu}, \boldsymbol{\Sigma})$. One way to broaden this parametric family for potential outliers is to adopt the two-component normal mixture p.d.f.

$$
(1-\epsilon) \phi(\boldsymbol{x} ; \boldsymbol{\mu}, \boldsymbol{\Sigma})+\epsilon \phi(\boldsymbol{x} ; \boldsymbol{\mu}, \boldsymbol{c} \boldsymbol{\Sigma}),
$$

where $c$ is large and $\epsilon$ is small, representing the small proportion of observations that have a relatively large variance. Huber (1964) subsequently considered more general forms of contamination of the normal distribution in the development of his robust $M$-estimators of a location parameter, as discussed further in Section 7. The normal scale mixture model (2) can be written as

$$
\int \phi(\boldsymbol{x} ; \boldsymbol{\mu}, \boldsymbol{\Sigma} / u) d H(u),
$$

where $H$ is the probability distribution that places mass $(1-\epsilon)$ at the point $u=1$ and mass $\epsilon$ at the point $u=1 / c$. Suppose we now replace $H$ by the p.d.f. of the square root of a chi-squared random variable on its degrees of freedom $\nu$; that is, by the random variable $U$ distributed as

$$
U \sim \operatorname{gamma}\left(\frac{1}{2} \nu, \frac{1}{2} \nu\right),
$$

where the gamma $(\alpha, \beta)$ density function $f(u ; \alpha, \beta)$ is given by

$$
f(u ; \alpha, \beta)=\left\{\beta^{\alpha} u^{\alpha-1} / \Gamma(\alpha)\right\} \exp (-\beta u) I_{(0, \infty)}(u) ; \quad(\alpha, \beta>0),
$$

and the indicator function $I_{(0, \infty)}(u)=1$ for $u>0$ and is zero elsewhere. We then obtain the $t$-distribution with location parameter $\mu$, positive definite inner product matrix $\Sigma$, and $\nu$ degrees of freedom,

$$
f(\boldsymbol{x} ; \boldsymbol{\mu}, \boldsymbol{\Sigma}, \nu)=\frac{\Gamma\left(\frac{\nu+p}{2}\right)|\boldsymbol{\Sigma}|^{-1 / 2}}{(\pi \nu)^{\frac{1}{2} p} \Gamma\left(\frac{\nu}{2}\right)\{1+\delta(\boldsymbol{x}, \boldsymbol{\mu} ; \boldsymbol{\Sigma}) / \nu\}^{\frac{1}{2}(\nu+p)}},
$$

where

$$
\delta(\boldsymbol{x}, \boldsymbol{\mu} ; \boldsymbol{\Sigma})=(\boldsymbol{x}-\boldsymbol{\mu})^{T} \boldsymbol{\Sigma}^{-1}(\boldsymbol{x}-\boldsymbol{\mu})
$$

denotes the Mahalanobis squared distance between $\boldsymbol{x}$ and $\boldsymbol{\mu}$ (with $\boldsymbol{\Sigma}$ as the covariance matrix). As $\nu$ tends to infinity, $U$ converges to one with probability one, and so $\boldsymbol{X}$ becomes marginally multivariate normal with mean $\boldsymbol{\mu}$ and covariance matrix $\boldsymbol{\Sigma}$. The family of $t$-distributions provides a heavy-tailed alternative to the normal family with mean $\boldsymbol{\mu}$ and covariance matrix that is equal to a scalar multiple of $\Sigma$ (if $\nu>2$ ). In the above and sequel, we are using $f$ as a generic symbol for a p.d.f. 


\section{ML Estimation of Mixtures of $t$-Distributions}

We consider now ML estimation for a $g$-component mixture of $t$-distributions, given by

$$
f(\boldsymbol{x} ; \boldsymbol{\Psi})=\sum_{i=1}^{g} \pi_{i} f\left(\boldsymbol{x} ; \boldsymbol{\mu}_{i}, \boldsymbol{\Sigma}_{i}, \nu_{i}\right)
$$

where now $\boldsymbol{\Psi}=\left(\pi_{1}, \ldots, \pi_{g-1}, \theta^{T}, \nu^{T}\right)^{T}$ and $\boldsymbol{\nu}=\left(\nu_{1}, \ldots, \nu_{g}\right)^{T}$. The application of the EM algorithm for ML estimation in the case of a single component $t$-distribution has been described in McLachlan and Krishnan (1997, Sections 2.6 and 5.8). The results there can be extended to cover the present case of a $g$-component mixture of multivariate $t$-distributions.

In the EM-framework, the observed data $\boldsymbol{x}_{1}, \ldots, \boldsymbol{x}_{n}$, are augmented by $z_{1}, \ldots, z_{n}$, where $z_{j}$ is the component-label vector defining the component of origin of $\boldsymbol{x}_{j}$, and $z_{i j}=\left(\boldsymbol{z}_{j}\right)_{i}$ is 1 or zero, according as $\boldsymbol{x}_{j}$ belongs or does not belong to the $i$ th component. In the light of the above characterization of the $t$-distribution, it is convenient to view the observed data augmented by the $z_{j}$ as still being incomplete and introduce into the complete-data vector the additional missing data, $u_{1}, \ldots, u_{n}$, which are defined so that given $z_{i j}=1$,

$$
\boldsymbol{X}_{j} \mid u_{j}, z_{i j}=1 \sim N\left(\boldsymbol{\mu}_{i}, \boldsymbol{\Sigma}_{i} / u_{j}\right)
$$

independently for $j=1, \ldots, n$, and

$$
U_{j} \mid z_{i j}=1 \sim \operatorname{gamma}\left(\frac{1}{2} \nu_{i}, \frac{1}{2} \nu_{i}\right) \text {. }
$$

Given $z_{1}, \ldots, z_{n}$, the $U_{1}, \ldots, U_{n}$ are independently distributed according to (8).

It follows that on the $(k+1)$ th iteration of the M-step, the $\boldsymbol{\mu}_{i}$ and $\boldsymbol{\Sigma}_{i}$ are updated as

$$
\boldsymbol{\mu}_{i}^{(k+1)}=\sum_{j=1}^{n} \tau_{i j}^{(k)} u_{i j}^{(k)} \boldsymbol{x}_{j} / \sum_{j=1}^{n} \tau_{i j}^{(k)} u_{i j}^{(k)}
$$

and

$$
\boldsymbol{\Sigma}_{i}^{(k+1)}=\sum_{j=1}^{n} \tau_{i j}^{(k)} u_{i j}^{(k)}\left(\boldsymbol{x}_{j}-\boldsymbol{\mu}_{i}^{(k+1)}\right)\left(\boldsymbol{x}_{j}-\boldsymbol{\mu}_{i}^{(k+1)}\right)^{T} / \sum_{j=1}^{n} \tau_{i j}^{(k)}
$$

where

$$
u_{i j}^{(k)}=\frac{\nu_{i}^{(k)}+p}{\nu_{i}^{(k)}+\delta\left(\boldsymbol{x}_{j}, \mu_{i}^{(k)} ; \boldsymbol{\Sigma}_{i}^{(k)}\right)}
$$

and where

$$
\tau_{i j}^{(k)}=\frac{\pi_{i}^{(k)} f\left(\boldsymbol{x}_{j} ; \boldsymbol{\mu}_{i}^{(k)}, \boldsymbol{\Sigma}_{i}^{(k)}, \nu_{i}^{(k)}\right)}{f\left(\boldsymbol{x}_{j} ; \boldsymbol{\Psi}^{(k)}\right)}
$$

is the current estimate of the posterior probability that $\boldsymbol{x}_{j}$ belongs to the $i$ th component of the mixture $(i=1, \ldots, g ; j=1, \ldots, n)$. It can be seen that the $\boldsymbol{\mu}_{i}^{(k+1)}$ and $\boldsymbol{\Sigma}_{i}^{(k+1)}$ are effectively chosen by weighted least-squares estimation. 
The E-step updates the weights $\tau_{i}^{(k)} u_{i j}^{(k)}$, while the M-step effectively chooses $\boldsymbol{\mu}_{i}^{(k+1)}$ and $\boldsymbol{\Sigma}_{i}^{(k+1)}$ by weighted least-squares estimation. Thus the updates of the parameters are available in closed form if the degrees of freedom $\nu_{i}$ are specified in advance. Otherwise, the updated $\nu_{i}^{(k+1)}$ have to be computed iteratively. The process can be speeded up using the multicycle ECM and ECME algorithms (Liu and Rubin (1995), McLachlan and Krishnan (1997, Section 5.8)). The MIXFIT algorithm of McLachlan et al. (1997) for the fitting of normal mixture models has an option for the fitting of mixtures of $t$-components.

\section{Clustering Applications of $t$-Mixture Models}

The ML estimation of the component means $\mu_{i}$ is robust in the sense that observations with large Mahalanobis distances are downweighted. This can be clearly seen from the form of the equation (9) for the MLE of $\mu_{i}$. As $\nu_{i}$ (or its estimate if not specified) decreases, the degree of downweighting of an outlier increases. For finite $\nu_{i}$ as $\left\|x_{j}\right\| \rightarrow \infty$, its effect on the $i$ th component-location parameter estimate goes to zero, whereas its effect on the $i$ th component-scale estimate remains bounded but does not vanish. Of course there is always the option of manually excluding observations considered to be grossly atypical of the bulk of the data, using the minimum covariance determinant criterion; see, for example, Hawkins and McLachlan (1997).

It can be therefore seen that the use of mixtures of $t$-distributions provides a sound statistical basis for formalizing and implementing the somewhat ad hoc approaches that have been proposed in the past. It also provides a framework for assessing the degree of robustness to be incorporated into the fitting of the mixture model through either the specification or the estimation of the degrees of freedom $\nu_{i}$ in the $t$-component p.d.f.'s.

\section{$6 \quad$ Example}

To illustrate the $t$-mixture model-based approach to clustering, we consider the crab data set of Campbell and Mahon (1974) on the genus Leptograpsus, which has been analysed further in Ripley (1996). Attention is focussed on the sample of $n=100$ blue crabs, there being $n_{1}=50$ males and $n_{2}=50$ females corre sponding to groups $G_{1}$ and $G_{2}$ respectively. Each specimen has measurements on the width of the frontal lip $F L$, the rear width $R W$, and length along the midline $C L$ and the maximum width $C W$ of the carapace, and the body depth $B D$ in mm. In Fig. 1, we give the scatter plot of the second and third variates with their group of origin noted. Hawkins' (1981) simultaneous test for multivariate normality and equal covariance matrices (homoscedasticity) suggests it is reasonable to assume that the group-conditional distributions are normal with a common covariance matrix. Consistent with this, it was found that the sample linear discriminant function (formed using the known classification of the data) misallocates only two observations (from $G_{1}$ ). 
We now cluster these data, ignoring the known classification of the data. We first fit a mixture of two normal components with equal covariance matrices. The implied clusters consist of one cluster containing 31 observations from $G_{1}$ and another containing all 50 observations from $G_{2}$ plus the remaining 19 observations from $G_{1}$. Hence this clustering misallocates 19 observations from $G_{1}$ but no observations from $G_{2}$. We next fitted a mixture of two $t$-components with common inner product matrix and degrees of freedom. The latter was estimated along with the other parameters from the data. The inferred value for $\nu$ was $\hat{\nu}=22.5$. This $t$-mixture model-based solution produces a slightly improved outright clustering in that one fewer observation from $G_{1}$ is misallocated with the smaller sized cluster now containing 32 observations from $G_{1}$ and with the larger one containing 18 observations from $G_{1}$ and all 50 from $G_{2}$.

Although the use of the $t$-mixture model has only slightly improved the outright clustering, it does produce a less extreme probabilistic clustering of the observations. To demonstrate this point, we have listed the estimates of the posterior probabilities of membership of group $G_{1}$ under both normal and $t$ mixture models for those 19 observations from $G_{1}$ misclassified under the normal mixture model in Table 1 . It can be seen that the use of the $t$-mixture model results in observation 14 having an estimated posterior probability of belonging to the first component of the mixture (corresponding to group $G_{1}$ ) of greater than 0.5 . Hence this observation is no longer misclassified in an outright clustering of the data. The remaining 18 observations would still be misclassified, but all have increased estimated posterior probabilities of belonging to $G_{1}$; in particular, these estimated probabilities for observations 11,18 , and 26 are much closer to the threshold value of 0.5 .

\section{Previous Work on $M$-Estimation of Components}

A common way in which robust fitting of normal mixture models has been undertaken, is by using M-estimates to update the component estimates on the M-step of the EM algorithm, as in McLachlan and Basford (1988) and Campbell (1994). In this case, the updated component means are $\mu_{i}^{(k+1)}$ are given by (9), but where now the weights $u_{i j}^{(k)}$ are defined as

$$
u_{i j}^{(k)}=\psi\left(d_{i j}^{(k)}\right) / d_{i j}^{(k)},
$$

where

$$
d_{i j}^{(k)}=\left\{\left(\boldsymbol{x}_{j}-\boldsymbol{\mu}_{i}^{(k)}\right)^{T} \boldsymbol{\Sigma}_{i}^{(k)^{-1}}\left(\boldsymbol{x}_{j}-\boldsymbol{\mu}_{i}^{(k)}\right)\right\}^{1 / 2}
$$

and $\psi(s)=-\psi(-s)$ is Huber's (1964) $\psi$-function defined by

$$
\begin{aligned}
\psi(s) & =s, & & |s| \leq c \\
& =\operatorname{sign}(s) c, & & |s|>c,
\end{aligned}
$$

for an appropriate choice of the tuning constant $c$. The $i$ th component-covariance matrix $\Sigma_{i}^{(k+1)}$ can be updated as $(10)$, where $u_{i j}^{(k)}$ is replaced by $\left\{\psi\left(d_{i j}^{(k)}\right) / d_{i j}^{(k)}\right\}^{2}$. 
An alternative to Huber's $\psi$-function is a redescending $\psi$-function, for example, Hampel's (1973) piecewise linear function. However, there can be problems in forming the posterior probabilities of component membership. as there is the question as to which parametric family to use for the component p.d.f.'s (McLachlan and Basford, Section 2.8, 1988). One possibility is to use the form of the p.d.f. corresponding to the $\psi$-function adopted. However, in the case of any redescending $\psi$-function with finite rejection points, there is no corresponding p.d.f. In Campbell (1994), the normal p.d.f. was used, while in the related univariate work in De Veaux and Kreiger (1990), the $t$-density with three degrees of freedom was used, with the location and scale component parameters estimated by the (weighted) median and mean absolute deviation, respectively. It can be therefore seen that the use of mixtures of $t$-distributions provides a sound statistical basis for formalizing and implementing the somewhat ad hoc approaches that have been proposed in the past. It also provides a framework for assessing the degree of robustness to be incorporated into the fitting of the mixture model through the specification or estimation of the degrees of freedom $\nu_{i}$ in the $t$-component p.d.f.'s.

\section{References}

Campbell, N.A. (1994). Mixture models and atypical values. Mathematical Geology $16,465-477$.

Campbell, N.A. and Mahon, R.J. (1974). A multivariate study of variation in two species of rock crab of genus Leptograpsus. Australian Journal of Zoology $22,417-425$.

Davé, R.N. and Krishnapuram, R. (1995). Robust clustering methods: a unified view. IEEE Transactions on Fuzzy Systems 5, 270-293.

Dempster, A.P., Laird, N.M., and and Rubin, D.B. (1977). Maximum likelihood from incomplete data via the EM algorithm (with discussion). Journal of the Royal Statistical Society $B$ 39, 1-38.

De Veaux, R.D. and Kreiger, A.M. (1990). Robust estimation of a normal mixture. Statistics \& Probability Letters 10, 1-7.

Frigui, H. and Krishnapuram, R. (1996). A robust algorithm for automatic extraction of an unknown number of clusters from noisy data. Pattern Recognition Letters 17, 1223-1232.

Hawkins, D.M. (1981). A new test for multivariate normality and homoscedasticity. Technometrics 23, 105-110. 
Hampel, F.R. (1973). Robust estimation: a condensed partial survey. $Z$. Wahrscheinlickeitstheorie verw. Gebiete 27, 87-104.

Hawkins, D.M. and McLachlan, G.J. (1997). High-breakdown linear discriminant analysis. Journal of the American Statistical Association 92, 136-143.

Huber, P.J. (1964). Robust estimation of a location parameter. Annals of Mathematical Statistics 35, 73-101.

Kharin, Y. (1996). Robustness in Statistical Pattern Recognition. Dordrecht: Kluwer.

Liu, C. and Rubin, D.B. (1995). ML estimation of the $t$ distribution using EM and its extensions, ECM and ECME. Statistica Sinica, 5, 19-39.

McLachlan, G.J. (1992). Discriminant Analysis and Statistical Pattern Recognition. New York: Wiley.

McLachlan, G.J. (1999). Finite Mixture Models. New York: Wiley.

McLachlan, G.J. and Basford, K.E. (1988). Mixture Models: Inference and Applications to Clustering. New York: Marcel Dekker.

McLachlan, G.J. and Krishnan, T. (1997). The EM Algorithm and Extensions. New York: Wiley.

McLachlan, G.J., Peel, D., Basford, K.E., and Adams, P. (1997). MIXFIT: an algorithm for the automatic fitting and testing of normal mixture models. Unpublished manuscript.

Ripley, B.D. (1996). Pattern Recognition and Neural Networks. Cambridge: Cambridge University Press.

Rocke, D.M. and Woodruff, D.L. (1997). Robust estimation of multivariate location and shape. Journal of Statistical Planning and Inference 57, 245-255.

Rousseeuw, P.J., Kaufman, L., and Trauwaert, E. (1996). Fuzzy clustering using scatter matrices. Computational Statistics and Data Analysis 23, 135-151.

Zhuang, X., Huang, Y., Palaniappan, K., and Zhao, Y. (1996). Gaussian density mixture modeling, decomposition and applications. IEEE Transactions on Image Processing 5, 1293-1302. 
Table 1. Estimated Posterior Probability of Membership of $G_{1}$

\begin{tabular}{|c|c|c|c|c|c|}
\hline No. & $\begin{array}{l}\text { Normal } \\
\text { Mixture }\end{array}$ & $\begin{array}{l}t \text {-Mixture } \\
\hat{\nu}=22.5\end{array}$ & No. & $\begin{array}{l}\text { Normal } \\
\text { Mixture }\end{array}$ & $\begin{array}{c}t \text {-Mixture } \\
\hat{\nu}=22.5\end{array}$ \\
\hline 1 & 0.0000 & 0.0004 & 11 & 0.1610 & 0.3237 \\
\hline 2 & 0.0000 & 0.0001 & 12 & 0.0042 & 0.0098 \\
\hline 3 & 0.0003 & 0.0010 & 14 & 0.4932 & 0.6359 \\
\hline 4 & 0.0016 & 0.0036 & 15 & 0.0116 & 0.0189 \\
\hline 5 & 0.0007 & 0.0020 & 16 & 0.0002 & 0.0003 \\
\hline 6 & 0.0056 & 0.0093 & 18 & 0.1702 & 0.2971 \\
\hline 7 & 0.0002 & 0.0005 & 19 & 0.0047 & 0.0068 \\
\hline 8 & 0.1450 & 0.1889 & 20 & 0.0733 & 0.0930 \\
\hline 9 & 0.0011 & 0.0022 & 26 & 0.4163 & 0.4643 \\
\hline 10 & 0.0004 & 0.0008 & & & \\
\hline
\end{tabular}

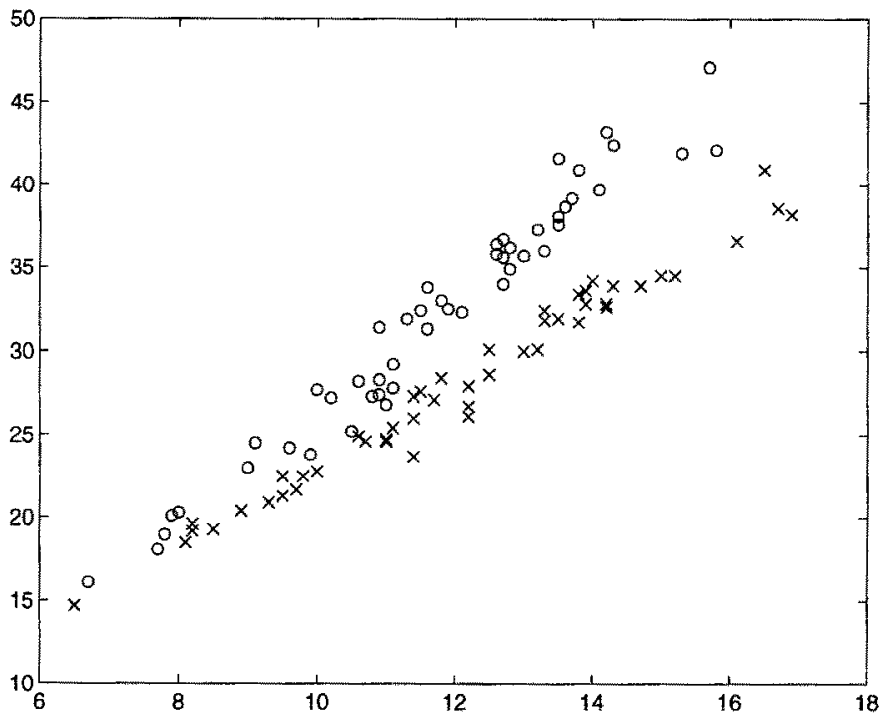

Fig. 1. Plot of third versus second variate for $n_{1}=50$ male and $n_{2}=50$ female "blue" crabs ( 0 denotes male and $x$ female) 